\title{
Double-Contrast Barium Enema Study With Computed Radiography: Assessment in Detection of Colorectal Polyps
}

\author{
Yoshitaka Okada, Shoichi Kusano, and Takashi Endo
}

Feasibility and diagnostic performance of digital radiography in gastrointestinal imaging have not been fully investigated. We performed double-contrast barium enema study with computed radiography (CRDCBE) on a routine basis, and the efficacy of this system for detection of colorectal polyps was investigated. The files of 76 patients, who had undergone both CR-DCBE and colonoscopy, were reviewed by two observers who were blinded to the colonoscopic findings. The radiation dose of CR-DCBE was reduced to $50 \%$ of conventional film-screen system. By using colonoscopic findings as a reference, the sensitivity and positive predictive value for colorectal polyps were $66 \%$ to $71 \%$ and $32 \%$ to $41 \%$, respectively. There was no significant interobserver difference. The use of postprocessing with gray-scale reversal and edge enhancement did not significantly improve the results. The sensitivity for polyps smaller than $1 \mathrm{~cm}$ was comparable with published data of conventional filmscreen systems. Our preliminary results show that CR-DCBE has an acceptable sensitivity for detection of colorectal polyps. It is suggested that the use of CR is a promising approach to digital gastrointestinal radiography.

Copyright o 1994 by W.B. Saunders Company

KEY WORDS: Digital radiography, double-contrast barium enema, colorectal polyp.

$\mathbf{T}$ HANKS TO the rapidly progressing computer technology, the development and clinical application of the picture archiving and communication system (PACS) is currently under active investigation. The conventional filmbased radiology is expected to be increasingly replaced by computer-based digital technology in the next few decades. In the PACS environment, all clinical images should be obtained, processed, and stored as digital data. Because digital radiography systems generally have limited spatial resolution compared with conventional film-screen radiography, a question has been raised whether digital radiography provides sufficient diagnostic information for clini-

From the Depanment of Radiology: Kitasato University East Hospital, Sagamihara, Japan.

Address reprint requests to Yoshitaka Okada, MD. Department of Radiology, Intemational Catholic Hospital, 2-5-1 Naka-ochiai, Shirjuku-ku. Tokyo 161. Japan.

Copyright 1994 by W.B. Saunders Company

0897-1889/94/0703-0003\$3.00/0 cal demands. A number of articles concerning the diagnostic performance of digital chest ${ }^{1-3}$ or bone ${ }^{4}$ radiography have been published. However, in the field of gastrointestinal radiology, evaluation of digital radiography has been limited. $5-7$

Digital radiography with storage-phosphor plate (computed radiography [CR]) is an evolving digital technique that has unique features including wide exposure latitude and extensive postprocessing capability. ${ }^{8.9}$ Since 1987 , we have been performing most double-contrast barium enema (DCBE) studies by routinely using CR. The aim of the present study was to assess the feasibility and diagnostic performance of DCBE with CR (CR-DCBE) for detection of colorectal polyps. We attempted to answer two questions: (1) whether CR-DCBE can detect colorectal polyps with acceptable sensitivity, and (2) whether the postprocessing with gray-scale reversal and edge enhancement provides any advantage in this setting.

\section{MATERIALS AND METHODS}

\section{Patients Population}

Between April 1987 and October 1990, ₹1,900 DCBE studies were performed with CR in our radiology department. By searching a computerized database, all patients that met the following two criteria were listed: (1) Proved or suspected diagnosis of colorectal polyp was registered in the database, and (2) both CR-DCBE and colonoscopy had been performed within a 1 -month period. Seventy-six patients met these criteria. Although the quality of CR-DCBE study was suboptimal in a few patients, no patients were excluded from the analysis. There were 46 men and 30 women ranging in age from 23 to 83 years (mean age. 55 years). According to the colonoscopy findings. 50 patients had one or more polyps, whereas 26 patients were eventually proved to be negative. A total of 70 polyps were found by colonoscopy (median size. $6 \mathrm{~mm}$ : range. 2 to $30 \mathrm{~mm}$ ).

\section{Technique of $C R-D C B E$}

C.R-DC.BE was done on remote-control equipment by residents and/or staff radiologists. Multiple spot radiographs were obtained at $90 \mathrm{kVp}$ by using 10 -in $\times 12$-in (25.4- $\mathrm{cm} \times 30.5-\mathrm{cm})$ storage-phosphor imaging plates instead of film-screen combination. The exposure dose was reduced to $50 \%$ of conventional film-screen DCBE.

The latent images on the imaging plates were read and digitized into $1.670-\times 2.510-\times 10$-bit $(1.024$ gray levels $)$ 
digital data with a TCR-201 CR system (Toshiba Medical. Tokyo, Japan). Two postprocessing afgorithms were routinely applied to each image. The first algorithm, the default mode. produces an image that simulates a conventional film-screen image. In the second algorithm, the reversal mode, the gray-scale polarity is reversed and moderate edge enhancement of high spatial frequencies (center of enhanced frequency range, $2.0 \mathrm{cycle} / \mathrm{mm}$ ) is applied. A pair of processed images for each exposure were displayed on a laser-printed hard copy by use of a 17.5- $\times 21.5-\mathrm{cm}$ film (CR633; Fuji Medical. Tokyo, Japan) with a two-on-one format.

\section{Obsener Experiment}

Two radiologists (S.K.. T.E.), who were unaware of the colonoscopy findings, independently reviewed the cases. Each case was reviewed twice by using different protocols. The reversal-mode image was masked and only the defaultmode image was reviewed in one session (protocol A), and both the default-mode and reversal-mode images were reviewed in the other session (protocol $B$ ). There was an interval of at least 3 weeks between the two readings of the same patient. The cases were presented in random order. and the observers recorded the location and size of all suspected polyps by filling in standard forms. Although no time constraint was imposed, the observers usually completed their evaluation in 4 to 6 minutes for each case.

\section{Data Analysis}

Sensitivity and positive predictive value for polyp detec tion were calculated for each observer by using colonoscopic findings as a standard of reference. The results were compared between the two observers and between the two protocols by means of McNemar test. ${ }^{10}$

This study was not designed as a comparative study between CR-DCBE and film-screen DCBE hecause it is impractical to perform both methods in every patient in a large clinical series. Hence, although cognizant of the limitation when making a comparison of the results from different study designs. we attempted to compare our sensitivity data with those of film-screen DCBE in the literature. We adopted the results of Rex et al because their data are assorted by polyp sizes. and therefore. ready to be compared with a different population. The polyps were classified by size ( 2 to $3 \mathrm{~mm}, 4$ to $5 \mathrm{~mm}, 6$ to $9 \mathrm{~mm}$, and $\geq 10 \mathrm{~mm}$ ). and the sensitivity for polyp detection was compared in each subgroup between our series and the data of Rex et al by using chi-square test or Fisher exact test.

The difference was considered significant if $P$ is smaller than 05 .

\section{RESULTS}

The sensitivity and positive predictive value for polyp detection by each observer were summarized in Table 1. The sensitivity was $66 \%$ to $71 \%$ and the positive predictive value was $32 \%$ to $41 \%$. Any difference between the two
Table 1. Sensitivity and Positive Predictive Value for Detection of Colorectal Polyps with CR-DCBE

\begin{tabular}{cccc}
\hline Observer & Protocol & Sensitivity & $\begin{array}{c}\text { Positive Predictive } \\
\text { Value }\end{array}$ \\
\hline 1 & A & 66 & 41 \\
& B & 71 & 39 \\
2 & A & 67 & 32 \\
& B & 67 & 32 \\
\hline
\end{tabular}

Numbers are percentages. Protocol A, only with defaultmode images. Protocol $B$, with both default-mode and reversalmode images.

observers or between protocol A and protocol B was not statistically significant $(P>.05$ by McNemar test).

The sensitivity data classified by polyp size are summarized in Table 2. The data of filmscreen DCBE reported by Rex et al ${ }^{11}$ are presented as well. The sensitivity for polyps larger than $10 \mathrm{~mm}$ in our series $(61 \%$ to $67 \%)$ was significantly lower than that in the series of Rex et al $(100 \%)(P<.05$ by chi-square test $)$. There were no significant differences between the two series in any other subgroups.

\section{DISCUSSION}

This study arises from an ongoing project for implementation of a large-scale digital radiology system in our 500-bed teaching hospital founded in 1986. Currently, in our radiology department, more than $90 \%$ of chest and bone radiographs and about $50 \%$ of special examinations (including gastrointestinal barium study, myelography, arthrography, intravenous urography, etc) are obtained with digital acquisition by using CR system. When an exposed imaging plate is read and digitized, the digital image data are transferred online to a digital image management system, where digital storage of all image data is executed. However, the digital storage is still performed on an experimental basis, and all permanent storage remains filmbased. The detail of this system has been published elsewhere. ${ }^{12}$

Despite many advantages of the computerbased digital technology, it still has not been widely used in gastrointestinal radiology, because the diagnostic value of digitally acquired barium studies has not been established. In this study, we evaluated the accuracy of CR-DCBE in detection of colorectal polyps, which are one of the most commonly encountered findings in 
Table 2. Sensitivity for Detection of Colorectal Polyps Classified by Sizes

\begin{tabular}{|c|c|c|c|c|c|c|}
\hline \multirow[b]{2}{*}{ Size $(\mathrm{mm})$} & & \multicolumn{2}{|c|}{ Observer 1} & \multicolumn{2}{|c|}{ Observer 2} & \multirow[b]{2}{*}{ Film-Screen DCBE } \\
\hline & & $\overline{\text { Protocol A }}$ & $\overline{\text { Protocol } \mathrm{B}}$ & Protocol A & Protocol B & \\
\hline $2-3$ & $(n=6)$ & 67 & 50 & 50 & 67 & 22 \\
\hline 4-5 & $(n=24)$ & 71 & 71 & 67 & 63 & 56 \\
\hline $6-9$ & $(n=22)$ & 68 & 82 & 77 & 77 & 83 \\
\hline$\geq 10$ & $(n=18)$ & $61^{*}$ & $67^{*}$ & $61^{*}$ & $67^{*}$ & 100 \\
\hline
\end{tabular}

Numbers are percentages.

*Significantly different from film-screen DCBE $(\rho<.05$ by chi-square test $)$.

practice. The sensitivity of film-screen DCBE for colorectal polyps has been reported to be $63 \%$ to $91 \% .^{11.13-16}$ To our knowledge, no data have been published concerning the colorectal polyp detection with digital radiography.

To compare our data with film-screen DCBE, we selected the data of Rex et al ${ }^{1 \prime}$ because of the following similarities in study design: (1) films were interpreted retrospectively by blinded observers, (2) colonoscopy was used as a standard of reference, and (3) the presented data were clearly assorted by polyp size. However, there are some significant differences between their study design and ours: (1) endoscopically placed metallic clips were used in their study to designate locations to be evaluated, whereas such markers were not used in our series, and (2) only the left colon was evaluated in their study.

The comparison between the results of our study and those of Rex et al showed that CR-DCBE and film-screen DCBE have comparable sensitivities for detection of polyps smaller than $10 \mathrm{~mm}$. We believe that this result is not dependent on the skills of observers, because there was no significant interobserver difference between our two observers. It should be noted that CR-DCBE was obtained with a radiation dose reduced to $50 \%$ of film-screen DCBE. Such a dose reduction was enabled by the wide exposure latitude of the photostimulable phosphor plate of CR system. Besides, technical failures caused by underexposure or overexposure seldom occurred in CR-DCBE, which was helpful in detecting some subtle abnormalities (Fig 1). These results are in accordance with a study by Krug et al, ${ }^{\circ}$ which showed that CRDCBE with $50 \%$ dose reduction was diagnostically equivalent to film-screen DCBE in 120 patients with a variety of colonic diseases.

In our study, the sensitivity for polyps larger than $10 \mathrm{~mm}$ was unexpectedly poor $(61 \%$ to
$67 \%$ ) and was significantly inferior to the results of film-screen DCBE by Rex et al (100\%). A possible explanation for this result is that our observers may have excessively concentrated their attention on small polyps because they were instructed to detect only polyps and to neglect any other abnormalities. To confirm this explanation, we made retrospective review of the missed lesions. Although each observer missed six or seven (33\% to $39 \%$ ) of 18 polyps larger than $10 \mathrm{~mm}$, all but one of these missed lesions can be seen in retrospect with knowledge of colonoscopic findings (Fig 2). Therefore, we believe that the low sensitivity for large polyps can be attributed to the performance of observers and should not be regarded as an inherent problem of CR images.

Extensive postprocessing capability of digital image data is another major advantage of $\mathrm{CR}$ system. Although it was our subjective impression that lesions were more conspicuous in the reversal-mode images, the blinded observer experiment showed that the sensitivity for polyp detection was not significantly improved by adding the reversal mode. Because most polyps were sharply marginated, the edge enhancement by postprocessing was not crucial for their visualization. Krug et al also reported that. in their series of $120 \mathrm{CR}-\mathrm{DCBE}$ studies, edge enhancement did not provide further diagnostic information. ${ }^{6}$ However, the postprocessing may play a significant role when more subtle mucosal abnormalities are examined.

In our study, the rate of false-positive reading was relatively high, resulting in low positive predictive value $(32 \%$ to $41 \%)$. Most falsepositive lesions were considered to be caused by air bubbles or fecal debris. Such errors could have been avoided if the movability of the suspected lesion were confirmed by comparing multiple films that depicted the same portion of the colon. Because most CR-DCBE studies in 


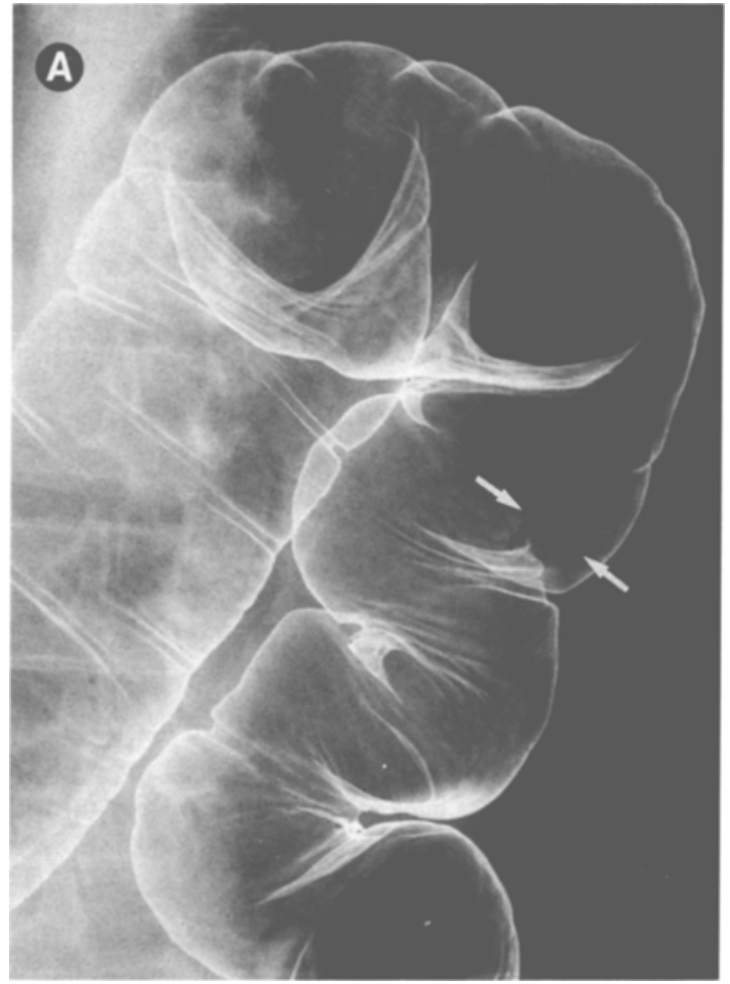

Fig 1. An 18-mm adenoma in the splenic flexure of a 38-year-old man. (A) An irregular-shaped flat elevation is barely visible on the default-mode image of CR-DCBE (arrow). (B) The reversal-mode image of CR-DCBE shows the lesion more conspicuously. $A$ granular appearance of the lesion can be better appreciated than in $A$. (C) $A$ radiograph of the film-screen DCBE study performed 12 months before $A$ and $B$. The lesion cannot be detected because of the overexposure in this portion of the colon.

this series had been performed by residents with various levels of skill, the high false-positive rate might be attributed, at least in part, to their failure to obtain constant-quality images of the entire colon. Nevertheless, we cannot exclude the possibility that some factors of CR system
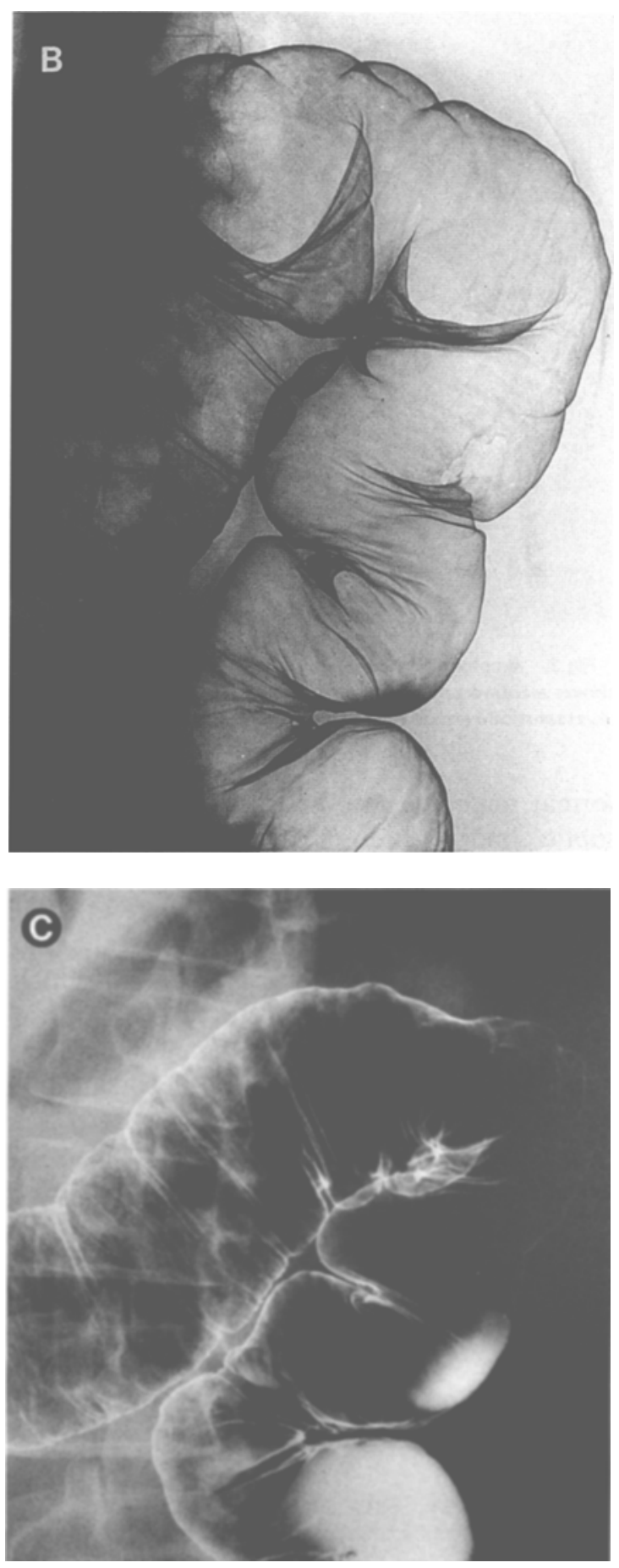

(eg, lower spatial resolution or smaller hardcopy display) might have adversely affected the observers' performance in differentiating truepositive and false-positive lesions. Several recent articles have suggested that the small hard-copy display of CR in standard two-on-one 

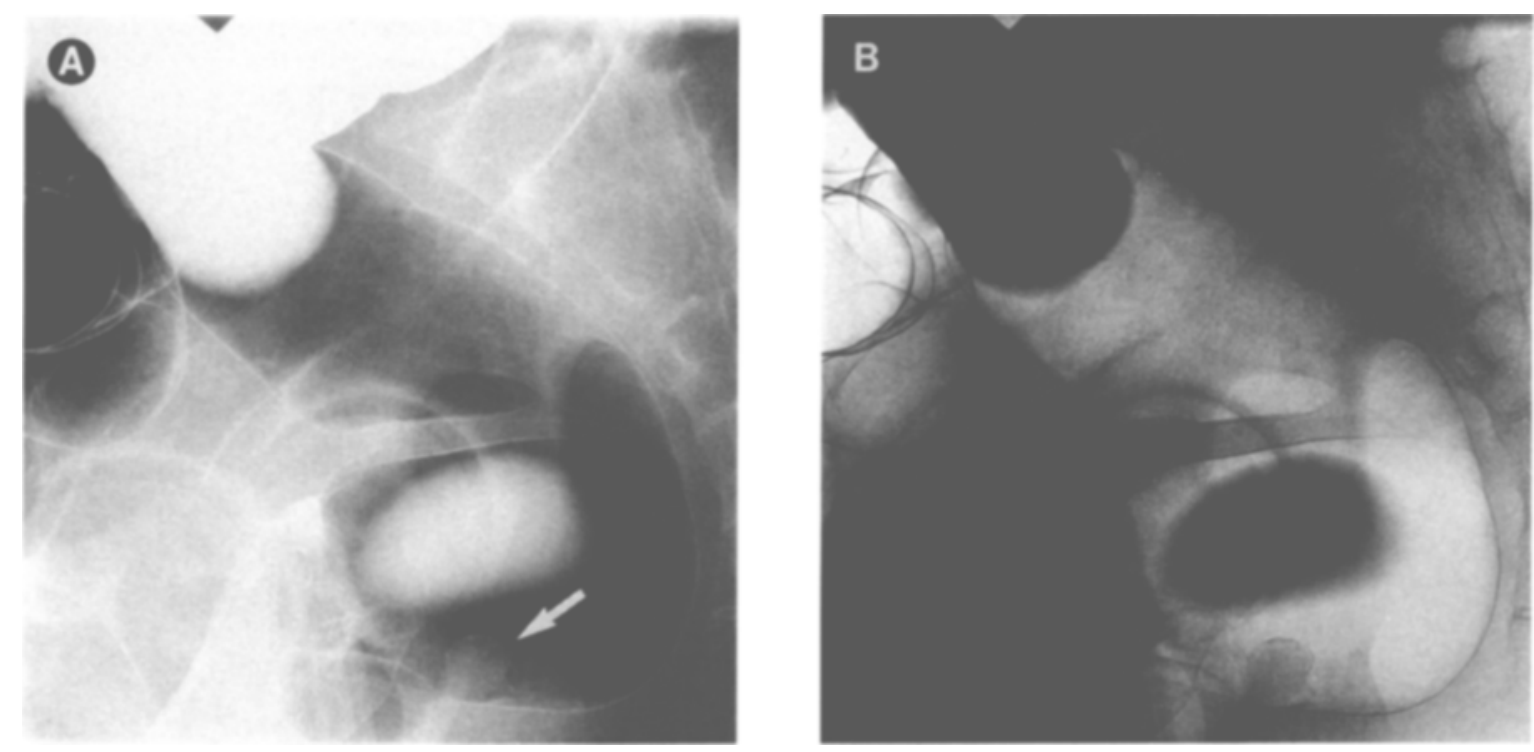

Fig 2. A colonoscopically confirmed 15-mm polyp in the rectum of a 64-year-old man. (A) The default-mode image of CR-DCBE shows a round polyp in the rectum (arrow). This lesion was missed by one observer in the blinded observer experiment. (B) The reversal-mode image of CR-DCBE. Both observers detected this lesion by adding this image.

format might be insufficient for evaluation of subtle abnormalities. ${ }^{17,18}$

The current study has several limitations. First, colonoscopy may not be a perfect standard, because it has been reported that the rate of polyps missed by colonoscopy may be as high as $10 \%$ to $20 \% .^{13-15}$ Therefore, the positive predictive value in this study may have been somewhat underestimated. The second problem is the work-up bias. Because this study was not prospectively controlled, most patients with negative CR-DCBE findings did not undergo colonoscopic confirmation. Because some of these patients may well have had undetected polyps, the sensitivity in this study may have been somewhat overestimated. Lastly, the comparison between CR-DCBE and film-screen DCBE cannot be regarded conclusive because this is not a direct comparative study of the two techniques.

In summary, this series showed that CR-
DCBE has an acceptable sensitivity for the detection of colorectal polyps. By comparison with film-screen DCBE, CR-DCBE enables reduction of radiation dose by $50 \%$. Because most of the chest and bone radiographs in our radiology department had already been replaced with CR, CR-DCBE was implemented with relatively small additional investment. Our initial experience suggests that the use of CR is a promising approach to a digital radiology department incorporating gastrointestinal barium studies. However, more extensive testing of the diagnostic value of CR-DCBE is certainly needed before it is widely accepted in clinical practice.

\section{ACKNOWLEDGMENT}

We thank the personnel of the Medical Record Department of our hospital for their assistance in preparing the case materials.

\section{REFERENCES}

1. MacMahon $\mathrm{H}$, Vyborny $\mathrm{CJ}$, Metz CE, et al: Digital radiography of subtle pulmonary abnormalities: An ROC study of the effect of pixel size on observer performance. Radiology 158:21-26, 1986

2. Schaefer CM, Greene R, Hall DA, et al: Mediastinal abnormalities: Detection with storage phosphor digital radiography. Radiology 178:169-173, 1991

3. Carr JJ, Reed JC, Choplin RH, et al: Plain and computed radiography for detecting experimentally induced pneumothorax in cadavers: Implications for detection in patients. Radiology 183:193-199, 1992

4. Wegryn SA, Piraino DW, Richmond BJ, et al: Comparison of digital and conventional musculoskeletal radiography: An observer performance study. Radiology 175:225228,1990

5. Kastan DJ, Ackerman LV, Feczko PJ: Digital gastroin- 
testinal imaging: The effect of pixel size on detection of subtle mucosal abnormalities. Radiology 162:853-856. 1987

6. Krug VB. Steinbrich W, Lorenz R. et al: Barium enema carried out by digital luminescent radiography (DLR) and conventional screen-film system combinations. ROFO 152:131-136. 1990 (abstr in English)

7. Takahashi M. Ueno S, Yoshimatsu S, et al: Gastrointestinal examinations with digital radiography. Radiographics 12:969-978, 1992

8. Sonoda M. Takano M. Miyahara J, et al: Computed radiography utilizing scanning laser stimulated luminescence. Radiology 148:833-838, 1983

9. Hilman BJ. Fajardo LL: Clinical assessment of phosphor-plate computed radiography: Equipment, strategy. and methods. J Digit Imaging 2:220)-227, 1989

10. Dwyer AJ: Matchmaking and McNemar in the comparison of diagnostic modalities. Radiology 178:328-330, !99!

11. Rex DK, Lehman GA. Lappas JC. et al: Sensitivity of double-contrast barium study for left-colon polyps. Radiology 158:69-72, 1986

12. Kusano S. Okada Y: Picture archiving and communi- cation system at the Kitasato University East Hospital: Promises and problems, in de Valk JPJ (ed): Integrated Diagnostic Imaging: digital PACS in medicine. Amsterdam, the Netherlands. Elsevier, 1992. pp 339-349

13. Thoeni RF. Menuck L: Comparison of barium enema and colonoscopy in the detection of small colonic polyps. Radiology 124:631-635. 1977

14. Fork F-T: Double contrast enema and colonoscopy in polyp detection. Gut 22:971-977, 1981

15. Thoeni RF. Petras A: Double-contrast bariumenema examination and endoscopy in the detection of polypoid lesions in the cecum and ascending colon. Radiology 144:257-260, 1982

16. Ott DJ, Chen YM. Gelfand DW, et al: Singlecontrast vs double-contrast barium enema in the detection of colonic polyps. Am J Roentgenol 146:993-996, 1986

17. Fisher PD, Brauer GW: Impact of image size on effectiveness of digital imaging systems. J Digit Imaging 2:39-41, 1989

18. Schaefer CM, Prokop M. Oestmann JW, et al: Impact of hard-copy size on observer performance in digital chest radiography. Radiology 184:77-81, 1992

\section{I want to join SCAR!}

Name:

Title:

Department:

Institution/Company:

\section{Membership category}

- Individual USA/Canada

- All other countries

- Student/Resident (Please provide letter from director.)

- Industrial sponsor
Business Address:

Phone:

Fax:

$\$ 125$ Please include payment in US dollars or charge by credit card.

Card No:

Exp. Date VISA MasterCard

Signature: 